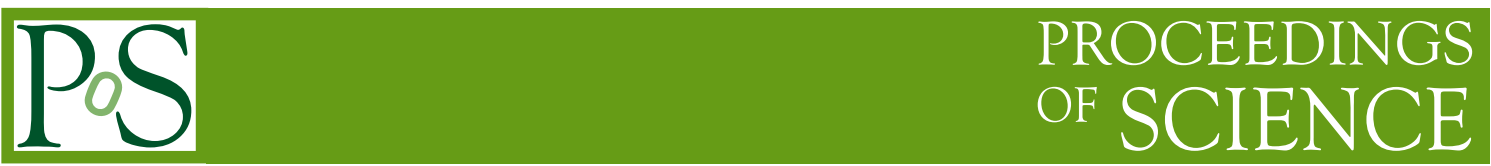

\title{
Correlations and textures in the neutrino mass matrix
}

\author{
Walter Grimus and Patrick Otto LudI* \\ University of Vienna, Faculty of Physics, Boltzmanngasse 5, A-1090 Vienna, Austria \\ E-mail: walter.grimus@univie.ac.at, patrick.ludl@univie.ac.at
}

\begin{abstract}
The recent enormous improvement of our knowledge of the neutrino oscillation parameters has motivated us to re-investigate the allowed ranges of the elements of the neutrino mass matrix in the basis where the charged-lepton mass mass matrix is diagonal. Moreover, we have studied the correlations of the elements of the neutrino mass matrix. The result of this analysis is useful for finding textures in the neutrino mass matrix and, therefore, for model building in the lepton sector. As an example, we present two textures of the neutrino mass matrix which have only two parameters and fit very well all current experimental data on the neutrino parameters.
\end{abstract}

The European Physical Society Conference on High Energy Physics

18-24 July, 2013

Stockholm, Sweden

\footnotetext{
* Speaker.
} 


\section{Correlations of the elements of the neutrino mass matrix}

Assuming Majorana neutrinos, in the basis where the charged-lepton mass matrix is diagonal, i.e. $\mathscr{M}_{\ell}=\operatorname{diag}\left(m_{e}, m_{\mu}, m_{\tau}\right)$, the mass matrix of the three light neutrinos is given by

$$
\mathscr{M}_{v}=U_{\mathrm{PMNS}}^{*} \operatorname{diag}\left(m_{1}, m_{2}, m_{3}\right) U_{\mathrm{PMNS}}^{\dagger} .
$$

Therefore, in this basis one finds that the absolute values of the elements of $\mathscr{M}_{v}$ depend on nine parameters, namely

$$
m_{0}, \Delta m_{21}^{2}, \Delta m_{31}^{2}, \theta_{12}, \theta_{23}, \theta_{13}, \delta, \rho, \sigma .
$$

Here $m_{0}$ denotes the mass of the lightest neutrino, the strongest bound on which comes from cosmology. In our analysis we used the upper bound $m_{0} \leq 0.3 \mathrm{eV}$. The allowed ranges for the six oscillation parameters were taken from the global fits of oscillation data of $[1,2]$. The phases $\rho$ and $\sigma$ denote the two experimentally unconstrained Majorana phases, which can thus assume any value between 0 and $2 \pi$.

Using the experimental input discussed above one can numerically derive restrictions on the absolute values $\left|\left(\mathscr{M}_{v}\right)_{i j}\right|$ of the elements of the neutrino mass matrix [3,4] and also compute correlations between these elements. We present two examples of correlation plots, ${ }^{1}$ both based on the global fit results of Forero et al. [1], assuming a normal spectrum. The boundaries of the corresponding $n \sigma$-regions are indicated as follows: best fit: $*, 1 \sigma: \mathbf{\Lambda}, 3 \sigma: \bullet$
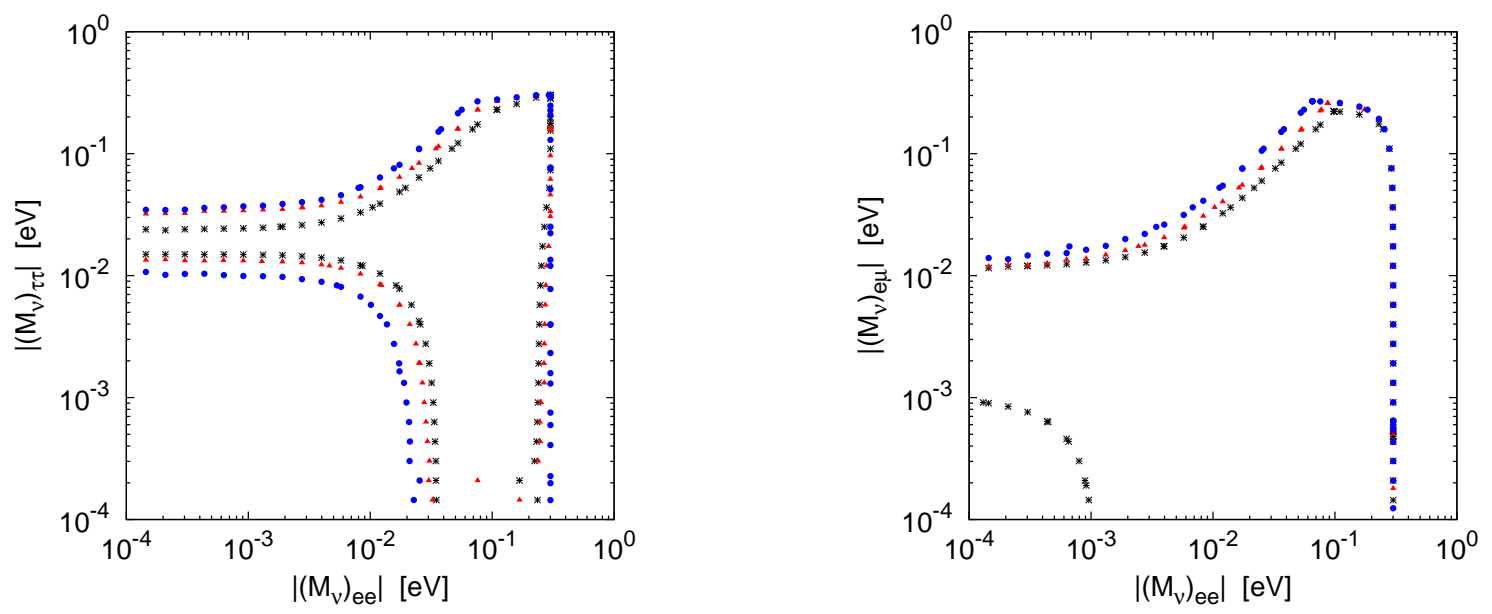

From the first plot we see that, even at the $3 \sigma$ level, there is a strong correlation between $\left|\left(\mathscr{M}_{v}\right)_{e e}\right|$ and $\left|\left(\mathscr{M}_{v}\right)_{\tau \tau}\right|$ which does not allow that the two matrix elements are small at the same time. In fact, all correlations we found to be stringent at the $3 \sigma$ level are of this type. The stringent correlations are the following ones:

$$
\begin{array}{llll}
\left|\left(\mathscr{M}_{v}\right)_{e e}\right| & \text { vs. } & \left|\left(\mathscr{M}_{v}\right)_{\mu \mu}\right| & \text { (normal spectrum) } \\
\left|\left(\mathscr{M}_{v}\right)_{e e}\right| & \text { vs. } & \left|\left(\mathscr{M}_{v}\right)_{\mu \tau}\right| & \text { (normal spectrum) } \\
\left|\left(\mathscr{M}_{v}\right)_{e e}\right| & \text { vs. } & \left|\left(\mathscr{M}_{v}\right)_{\tau \tau}\right| & \text { (normal spectrum) } \\
\left|\left(\mathscr{M}_{v}\right)_{\mu \mu}\right| & \text { vs. } & \left|\left(\mathscr{M}_{v}\right)_{\mu \tau}\right| & \text { (normal spectrum) } \\
\left|\left(\mathscr{M}_{v}\right)_{\mu \tau}\right| & \text { vs. } & \left|\left(\mathscr{M}_{v}\right)_{\tau \tau}\right| & \text { (normal spectrum). }
\end{array}
$$

\footnotetext{
${ }^{1}$ The light Majorana neutrino mass matrix has six independent entries, thus there are 15 correlations. Since there are two possible mass spectra, there is a total of 30 plots.
} 
For an inverted mass spectrum there are no correlations manifest at the $3 \sigma$ level.

Turning to the second plot we see that (in the case of a normal mass spectrum), at 1,2 and $3 \sigma$, $\left(\mathscr{M}_{v}\right)_{e e}$ and $\left(\mathscr{M}_{v}\right)_{e \mu}$ can simultaneously be very small, in contrast to the first plot. This consideration leads to the concept of texture zeros in the neutrino mass matrix [5]. Since they are easily realizable by means of Abelian flavour symmetries [6], texture zeros have become very popular in model building. An obvious question arising in this context is whether the neutrino mass matrix supports more structure that just texture zeros, i.e., whether one can impose additional relations leading to so-called "hybrid textures."

\section{Hybrid textures of the neutrino mass matrix}

Our strategy to unveil possible "hybrid textures" of $\mathscr{M}_{v}$ is the following [7]. We define the function

$$
F_{i j k l} \equiv\left|\left(\mathscr{M}_{v}\right)_{i j}\right|+\left|\left(\mathscr{M}_{v}\right)_{k l}\right|,
$$

which can be minimized within the allowed range of its nine parameters-see equation (1.2). A minimum of zero (within the numerical accuracy) displays an allowed case of two texture zeros. This analysis does not only confirm the results deduced from the correlation plots of section 1, it also provides an estimate of the neutrino mass matrix in a certain sense. Namely, taking the nine parameters at the minimum of $F_{i j k l}$, we can estimate the values of $\left|\left(\mathscr{M}_{v}\right)_{i j}\right|$ for all $i, j=e, \mu, \tau$. In this way we find two types of two texture zeros which can be endowed with a hybrid structure, namely [7]

$$
\mathscr{M}_{v}=\left(\begin{array}{ccc}
0 & 0 & a \\
0 & b & 2 a \\
a & 2 a & b
\end{array}\right) \quad \text { and } \quad \mathscr{M}_{v}=\left(\begin{array}{ccc}
0 & a & 0 \\
a & b & 2 a \\
0 & 2 a & b
\end{array}\right) .
$$

Surprisingly, the above textures are very well compatible with observations even for real parameters $a$ and $b$. In this case, the absolute neutrino mass scale, the three mixing angles and the two masssquared differences are functions of only two real parameters.

Acknowledgments: This work is supported by the Austrian Science Fund (FWF), Project No. P 24161-N16.

\section{References}

[1] D.V. Forero, M. Tortola and J.W.F. Valle, Phys. Rev. D 86 (2012) 073012 [arXiv:1205.4018].

[2] G.L. Fogli, E. Lisi, A. Marrone, D. Montanino, A. Palazzo and A.M. Rotunno, Phys. Rev. D 86 (2012) 013012 [arXiv:1205.5254].

[3] A. Merle and W. Rodejohann, Phys. Rev. D 73 (2006) 073012 [hep-ph/0603111].

[4] W. Grimus and P.O. Ludl, JHEP 1212 (2012) 117 [arXiv:1209.2601].

[5] P.H. Frampton, S.L. Glashow and D. Marfatia, Phys. Lett. B 536 (2002) 79 [hep-ph/0201008].

[6] W. Grimus, A.S. Joshipura, L. Lavoura and M. Tanimoto, Eur. Phys. J. C 36 (2004) 227 [hep-ph/0405016].

[7] W. Grimus and P.O. Ludl, J. Phys. G 40 (2013) 055003 [arXiv:1208.4515]. 